\title{
Estado atual da cirurgia de revascularização do miocárdio
}

\author{
Coronary artery bypass graft: \\ state of the art
}

\author{
Paulo M. Pêgo-Fernandes' ${ }^{1}$, Fabio A. Gaiotto², \\ Flávio Guimarães-Fernandes ${ }^{3}$
}

\begin{abstract}
Pego-Fernandes PM, Gaiotto FA, Guimarães-Fernandes F. Estado atual da cirurgia de revascularização do miocárdio. Rev Med (São Paulo). 2008 abr.-jun.;87(2):92-8.

RESUMO: A doença aterosclerótica cardiovascular é a maior causa de morte tanto no Brasil quanto nos países desenvolvidos, sendo, portanto, um assunto de relevância tanto para profissionais da área de saúde quanto para a população geral. Apresentamos, então, as formas de tratamento da doença, principalmente no enfoque cirúrgico, com a revascularização do miocárdio. Para tanto, iniciamos com um pequeno histórico que nos mostra como se chegou à operação como conhecemos hoje. Discutimos as técnicas atualmente empregadas neste tipo de operação e quando ela é feita com ou sem circulação extra-corpórea; quais são os pacientes com indicação para esse tipo de tratamento e o que se espera dos resultados, quais as perspectivas desse procedimento; e como a prática de cirurgias minimamente invasivas e a utilização da robótica auxiliam o cirurgião. Por fim, contextualizamos dois procedimentos novos: a revascularização percutânea, que ainda apresenta resultados incertos a longo prazo e a neoangiogênese, que é um procedimento em fase de estudo.
\end{abstract}

DESCRITORES: Revascularização miocárdica. Isquemia miocárdica/cirurgia. Procedimentos cirúrgicos minimamente invasivos.

\section{INTRODUÇÃO}

A doença aterosclerótica cardiovascular é a maior causa de morte tanto no Brasil quanto nos países desenvolvidos, sendo responsável por $36 \%$ dos óbitos em pessoas com idade entre 50 e 64 anos e $42 \%$ em pessoas com idade superior a 65 anos $^{1}$. No coração, a doença aterosclerótica manifesta-se através da obstrução coronariana.

\footnotetext{
1. Professor Associado de Cirurgia Torácica e Cardiovascular do Departamento de Cardiopneumologia da FMUSP.

2. Doutor em Cirurgia Torácica pela FMUSP.

3. Acadêmico do segundo ano de Medicina da FMUSP.

Endereço para correspondência: Paulo M. Pêgo-Fernandes. Instituto do Coração da Faculdade de Medicina da Universidade de São Paulo. Avenida Dr. Enéias de Carvalho Aguiar, 44, Bloco II, 2º andar, sala 9. São Paulo, SP. e-mail: paulopego@incor.usp.br
} 
Pego-Fernandes PM et al. Estado atual da cirurgia de revascularização do miocárdio.

O quadro clínico da obstrução aterosclerótica coronariana é amplo e os sintomas são bastante diversificados. Esta doença pode ser classificada, de maneira simplificada, em insuficiência coronariana aguda e crônica, sendo que em ambas as formas a falta de sangue ao miocárdio é a causa da doença. O tratamento da insuficiência coronariana pode ser clínico, através de medicamentos e de mudanças nos hábitos de vida, ou cirúrgico, através de intervenções mais invasivas de revascularização do miocárdio².

O tratamento clínico apresenta bons resultados. Entretanto, quando há angina refratária, diminuição da força de contração do coração (ocasionando insuficiência cardíaca) e risco de vida, a intervenção de revascularização se faz necessária ${ }^{2}$. Todas as modalidades de revascularização do miocárdio têm como principal objetivo o reestabelecimento do fornecimento sanguíneo adequado ao miocárdio e, para isso, abordam o sistema de condução do sangue: as artérias coronárias ${ }^{12}$.

Nos últimos 10 anos, houve marcante avanço no tratamento clínico e nos procedimentos de revascularização do miocárdio. O emprego de antiagregantes plaquetários e as estatinas trouxeram melhorias ao tratamento clínico ${ }^{5}$. A cirurgia de revascularização do miocárdio passa por aprimoramento progressivo, visando à menor agressão ao paciente e o emprego de enxertos de maior durabilidade ${ }^{7}$.

Dentre as melhorias nas formas de intervenção de revascularização, vale destacar o advento da angioplastia (uma técnica que se utiliza de balão inflado dentro da artéria coronária obstruída) e dos stents (endoprótese expansível em forma de tubo para diminuir o risco de reestenose), que mudaram a forma de abordagem e melhororam o resultado do tratamento da síndrome coronariana aguda ${ }^{5}$; e a utilização das células tronco, que abre novas perspectivas médicas para o tratamento da insuficiência coronariana.

\section{CIRURGIA DE REVASCULARIZAÇÃO DO MIO- CÁRDIO}

\section{Histórico}

O miocárdio é um músculo de alta demanda de oxigênio e nutrientes, sendo as artérias coronárias as responsáveis por este fornecimento. Há grande reserva coronariana para a irrigação do miocárdio. Entretanto, com o progredir das obstruções, a falta de sangue deve ser revertida e a cirurgia cardíaca oferece a técnica cirúrgica de revascularização do miocárdio, a qual permite que o sangue seja transportado para o território isquêmico através de um conduto.

Em 1945, Vineberg propôs a revascularização do miocárdio de maneira indireta: implantando a artéria torácica interna esquerda no miocárdio isquêmico. Os resultados iniciais foram questionados, pois não havia como demonstrar o seu funcionamento, já que a angiografia (método de visualização dos vasos sanguíneos que, através da injeção de contraste e da utilização de raios $x$, permite que sejam detectadas anomalias, no caso, a obstrução coronariana) ainda não era disponível ${ }^{12}$.

A partir de 1959, com os estudos de Sones, na Cleveland Clinic, os exames de cateterismo cardíaco passaram para a prática clínica e o funcionamento do procedimento proposto por Vineberg foi demonstrado. O interesse pela revascularização do miocárdio tornouse, então, crescente ${ }^{12}$.

Em 1967, René Favaloro, cirurgião argentino que se aperfeiçoava na Cleveland Clinic, propôs a utilização da veia safena para a realização de uma anastomose direta no sistema coronariano ${ }^{10}$ (Figura 1). Paralelamente, na Rússia, Kolesov realizava as primeiras anastomoses de artéria torácica interna esquerda com ramos artéria coronária interventricular anterior ${ }^{11}$. Iniciava-se, assim, a popularização e emprego rotineiro do procedimento que, atualmente, é um dos maiores alvos de publicações e estudos da área médica: a revascularização do miocárdio.



Figura 1. Veia safena magna dissecada por mini-incisões

No Brasil, o Prof. Dr. Adib Jatene foi o pioneiro na operação de revascularização do miocárdio, sendo essa técnica utilizada atualmente em milhares de pacientes em nosso país.

\section{Circulação extra-corpórea}

Os primeiros procedimentos de revascularização do miocárdio eram feitos sem o emprego da circulação extra-corpórea. O advento dessa tecnologia e o avanço das técnicas anestésicas permitiram a ampliação do tratamento cirúrgico das obstruções coronarianas $^{12}$. 
O sistema de circulação extra-corpórea permite que o cirurgião trabalhe com o coração parado. Para isto, o sistema deve substituir por completo as funções de bombeamento e de oxigenação do sangue. As bombas sanguíneas podem ser de roletes ou centrífugas (Figura 2) e os oxigenadores mais empregados são os de membrana (Figura 3).

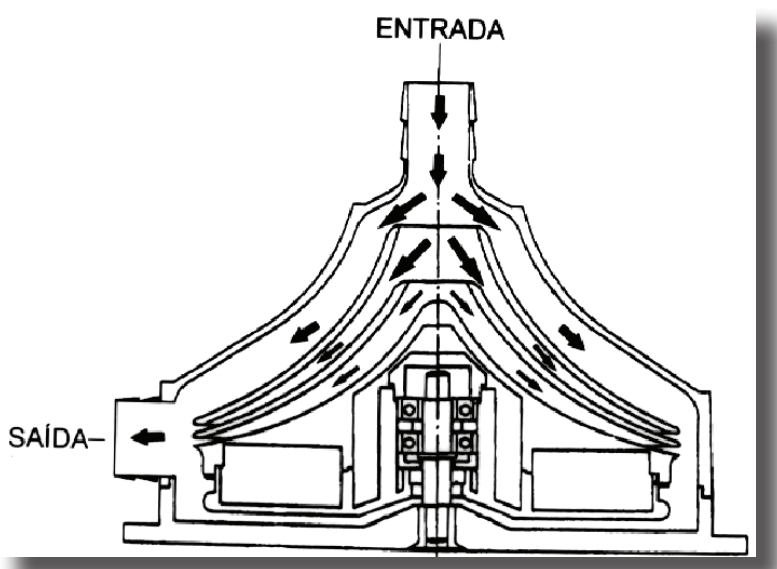

Figura 2. Esquema de bomba centrífuga

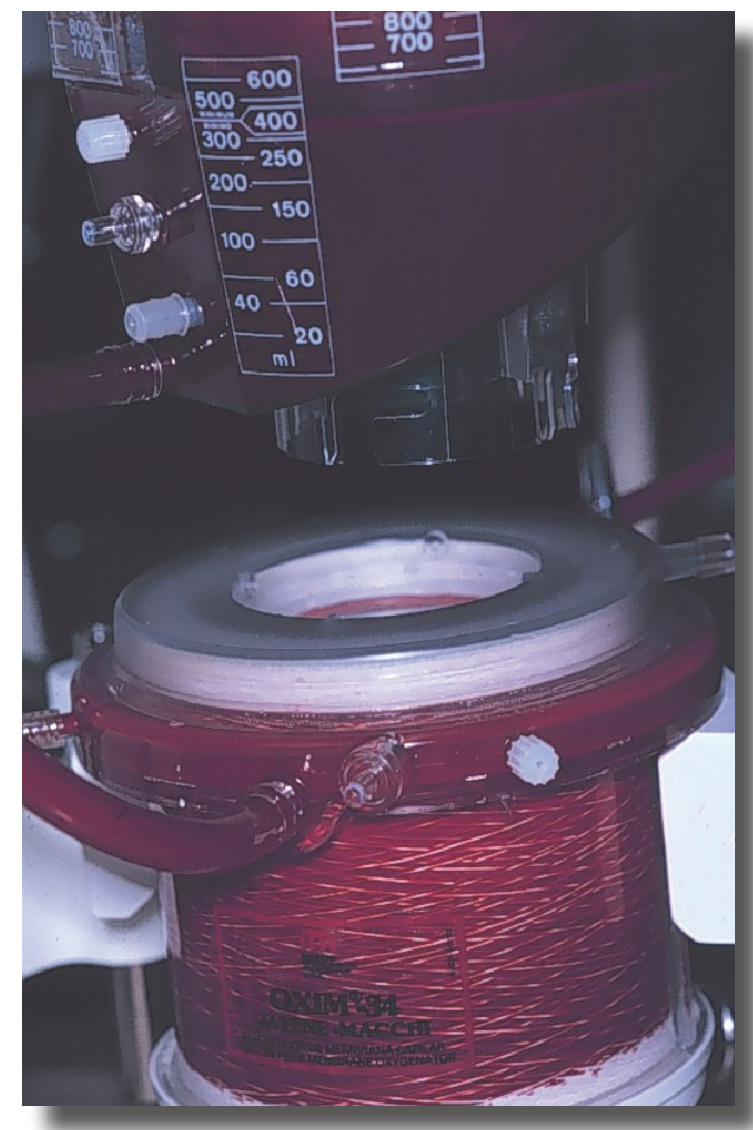

Figura 3. Oxigenador de membranas acoplado ao reservatório venoso
A circulação extra-corpórea permite o tratamento de doenças intra-cavitárias, sendo as valvopatias e as cardiopatias congênitas os grupos em que o seu emprego é quase imprescindível ${ }^{12}$. $\mathrm{Na}$ revascularização do miocárdio, há a possibilidade do tratamento cirúrgico ser realizado sem a utilização da circulação extra-corpórea ${ }^{1}$. corpórea

Revascularização sem circulação extra-

Os efeitos deletérios da circulação extracorpórea são importantes e, dentre eles, devemos destacar as coagulopatias e as disfunções transitórias dos pulmões, dos rins e do sistema nervoso central ${ }^{12}$. O desenvolvimento de estabilizadores de superfície - equipamentos de fácil manuseio que permitem a confecção de suturas com o coração batendo permitiu o aperfeiçoamento da revascularização do miocárdio sem circulação extra-corpórea.

Associando-se os estabilizadores às manobras de exposição (Figura 4) do coração e às táticas anestésicas apropriadas, todos os vasos podem ser abordados com o coração em funcionamento. $\mathrm{O}$ tratamento cirúrgico pode ser feito de maneira segura e confiável, sem os efeitos deletérios da circulação extra-corpórea ${ }^{1}$. Com a diminuição do trauma operatório, reduz-se o tempo de permanência na Unidade de Terapia Intensiva, o tempo de permanência no hospital e o custo total dos procedimentos.

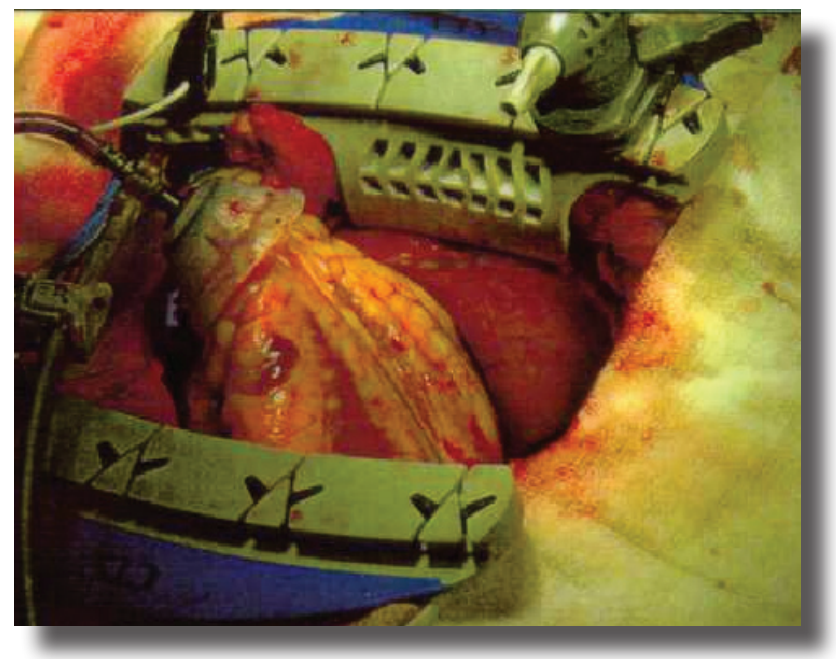

Figura 4. Sucção apical do coração favorecendo a exposição das coronárias na parede inferior

Idosos e portadores de insuficiência renal, de DPOC (Doença Pulmonar Obstrutiva Crônica), de disfunções neurológicas, de neoplasias e pacientes que não podem receber hemotransfusão são os que mais se beneficiam da revascularização sem circulação 
Pego-Fernandes PM et al. Estado atual da cirurgia de revascularização do miocárdio.

extra-corpórea. Entretanto, apesar das vantagens, muitos pacientes não podem ser operados desta maneira. Corações muito aumentados e sistema coronariano acometido por doenças difusas e por obstruções que promovem instabilidade hemodinâmica são, entre outros, fatores que impossibilitam o emprego da técnica ${ }^{9}$.

\section{Indicações e resultados}

A revascularização cirúrgica do miocárdio, sumariamente, está indicada para aqueles pacientes nos quais o tratamento clínico não consegue controlar a angina pectoris ou para aqueles que possuem um elevado grau de obstrução de artérias coronárias principais levando ao risco de vida. Geralmente são pacientes que apresentam lesões coronarianas difusas, com acometimento de mais de um território coronariano e com envolvimento de artérias vitais, como o tronco da coronária esquerda e o ramo descendente anterior ${ }^{5}$.

Nos portadores de síndrome coronariana aguda, após a abordagem percutânea da "artéria culpada", os outros ramos acometidos, se existir, devem ser tratados clínica ou cirurgicamente, após a fase aguda do infarto. Quando há complicação do infarto

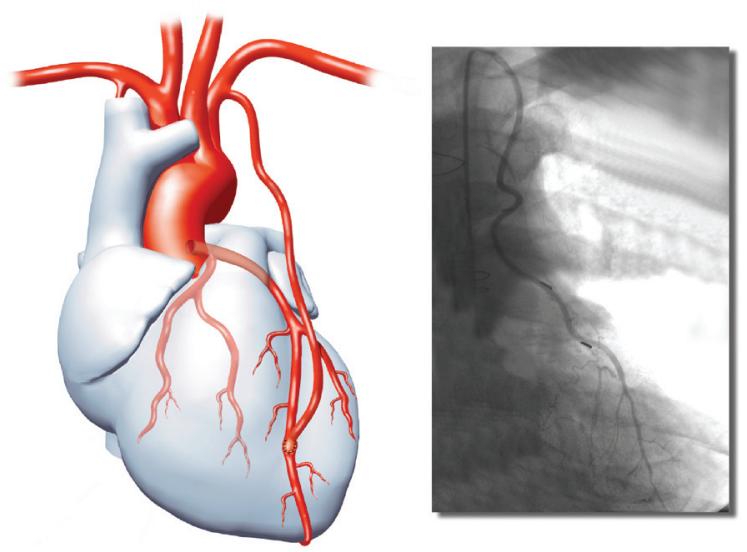

Figura 5A. Artéria torácica interna esquerda anastomosada com o ramo interventricular anterior e resultado angiográfico de 22 anos de seguimento

\section{Perspectivas}

O desenvolvimento de novas tecnologias e de técnicas operatórias tem proporcionado à cirurgia de revascularização do miocárdio um caráter menos invasivo: incisões menores; sem circulação extra-corpórea, sem manipulação da aorta, menor utilização de hemoderivados e utilização da robótica (Figura 6). Isto se traduz em menor trauma operató- agudo do miocárdio (comunicação interventricular pós-infarto, choque cardiogênico, ruptura ventricular, insuficiência mitral isquêmica) o tratamento cirúrgico deve ser realizado emergencialmente ${ }^{12}$.

Os resultados da revascularização cirúrgica do miocárdio são bem conhecidos e, indubitavelmente, melhoram a qualidade e a expectativa de vida dos pacientes ${ }^{14}$. O bom resultado depende não apenas da indicação adequada, mas também dos enxertos empregados. O emprego de, pelo menos, uma artéria torácica interna mostra grande impacto na sobrevida dos pacientes submetidos à revascularização do miocárdio?.

São dois os tipos de condutos empregados para a revascularização do miocárdio: condutos venosos e condutos arteriais. O conduto venoso mais utilizado é a veia safena magna, que apesar de ser de fácil utilização, apresenta degeneração progressiva ao longo do seguimento pós-operatório sendo que, ao final de 10 anos, apenas $30 \%$ das pontes estão pérvias $^{6}$. Os condutos arteriais, em especial as artérias torácicas internas, apresentam maior durabilidade, atingindo perviabilidade de $90 \%$ em 15 anos $^{6}$ (Figura 5A). A utilização de outras artérias (Figura 5B) deve ser estimulada por apresentarem maior durabilidade quando comparadas à veia safena.

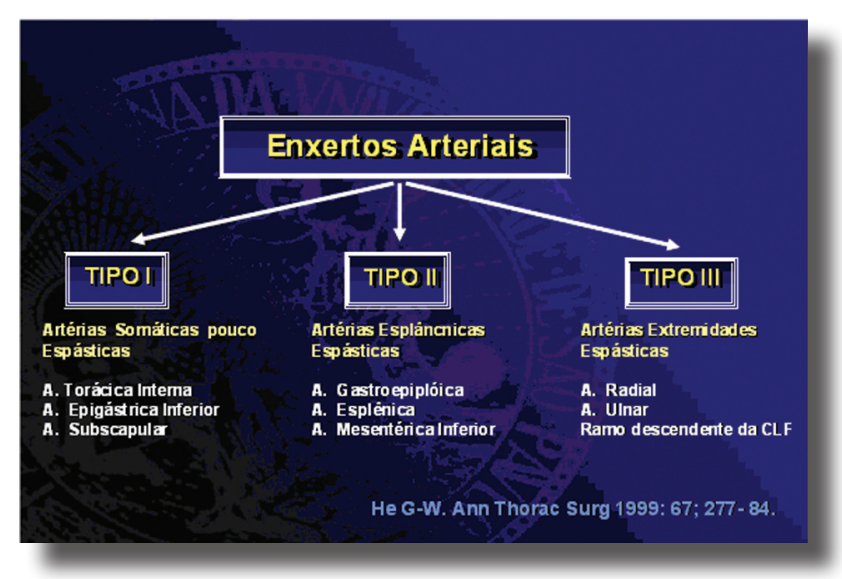

Figura 5B. Tipos de enxertos arteriais

rio e recuperação mais rápida do paciente.

O perfil dos pacientes mudou com o advento da revascularização percutânea e a melhora do tratamento clínico. Hoje, são operados os pacientes mais graves e que apresentam maior complexidade. É fundamental que o cirurgião esteja preparado para todas as situações de campo operatório, tentando descobrir e realizar a cirurgia ideal para cada paciente. 


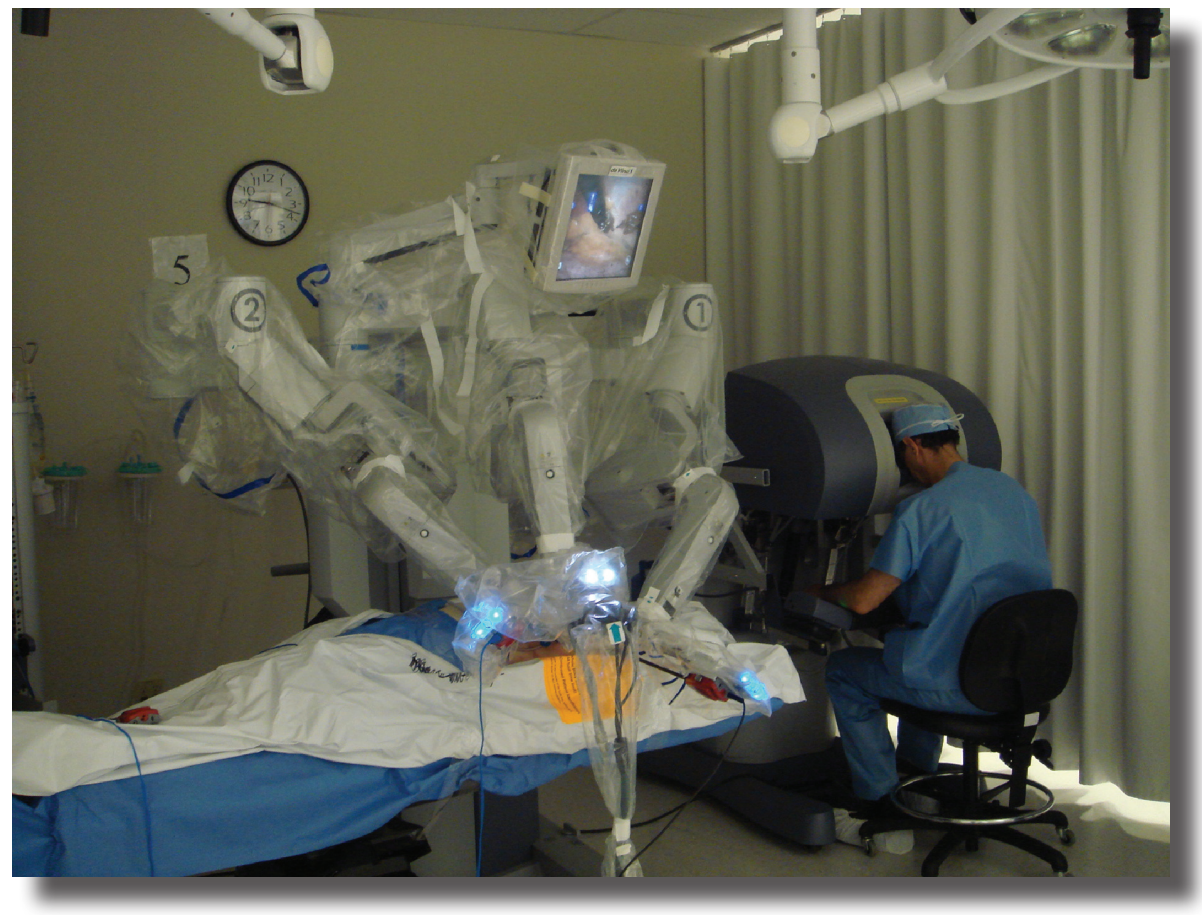

Figura 6. Cirurgia cardíaca com o emprego da robótica

A doença aterosclerótica do coração é responsável por uma parcela enorme dos óbitos em humanos ${ }^{1} \mathrm{e}$ isto não mudou com o desenvolvimento do tratamento percutâneo ou clínico. Houve melhora na longevidade e no controle dos sintomas.

O foco clínico deve ser a prevenção. Quando esta for eficaz, seja por intervenção medicamentosa ou por mudanças no estilo de vida, a doença aterosclerótica estará controlada e todos os métodos de revascularização do miocárdio estarão obsoletos. Infelizmente, há muito que se descobrir sobre a aterosclerose, pois a sua origem é multifatorial.

\section{CONCLUSÃO}

O método de revascularização do miocárdio, além de ser específico para cada paciente, não é uma decisão a ser tomada apenas por um profissional, mas sim em consenso com o paciente, clínico, cirurgião e hemodinamicista. O objetivo principal de qualquer método de revascularização do miocárdio deve ser a longa sobrevivência do seu paciente, com qualidade de vida satisfatória.

\section{Revascularização percutânea}

A cardiologia passou, nos últimos 10 anos, por grandes mudanças e os procedimentos de desobstru- ção percutânea das artérias coronárias, sem dúvida, merecem destaque especial.

Os primeiros procedimentos deste tipo eram feitos com dilatação das lesões através da insuflação de balão intra-coronariano. Rapidamente ocorria a reestenose e, então, desenvolveram-se os stents, que são malhas metálicas que emolduram o local da obstrução, evitando a retração elástica da placa aterosclerótica.

Os stents metálicos mostraram-se ineficazes a médio prazo, em virtude da proliferação neointimal (reação inflamatória ao corpo estranho dentro da artéria coronária levando a um aumento das células endoteliais) e reestenose. Desenvolveu-se, ainda, uma classe de medicamentos poderosos que atuam diminuindo a agregação plaquetária, diminuindo a chance de reestenose. Ainda, os stents revestidos com substâncias inibidoras da proliferação celular (stents farmacológicos) diminuíram mais ainda a chance de reestenose, porém mantém um alto risco de trombose ${ }^{13}$.

Os resultados a longo prazo são incertos e a terapia percutânea tem lugar marcado na sua indicação: síndrome coronariana aguda ${ }^{3}$. Nos quadros crônicos e estáveis, a escolha do método de revascularização deve respeitar cada paciente de forma individualizada e deve ser tomada em conjunto com o clínico, cirurgião e hemodinamicista. 
Pego-Fernandes PM et al. Estado atual da cirurgia de revascularização do miocárdio.

\section{Neoangiogênese}

A doença aterosclerótica pode atingir estágios avançados de evolução com o acometimento difuso de todas as coronárias. Quando não é possível a revascularização cirúrgica ou percutânea, surge uma nova opção que ainda apresenta resultados incertos: a neoangiogênese.

Nesta modalidade terapêutica, células pro-

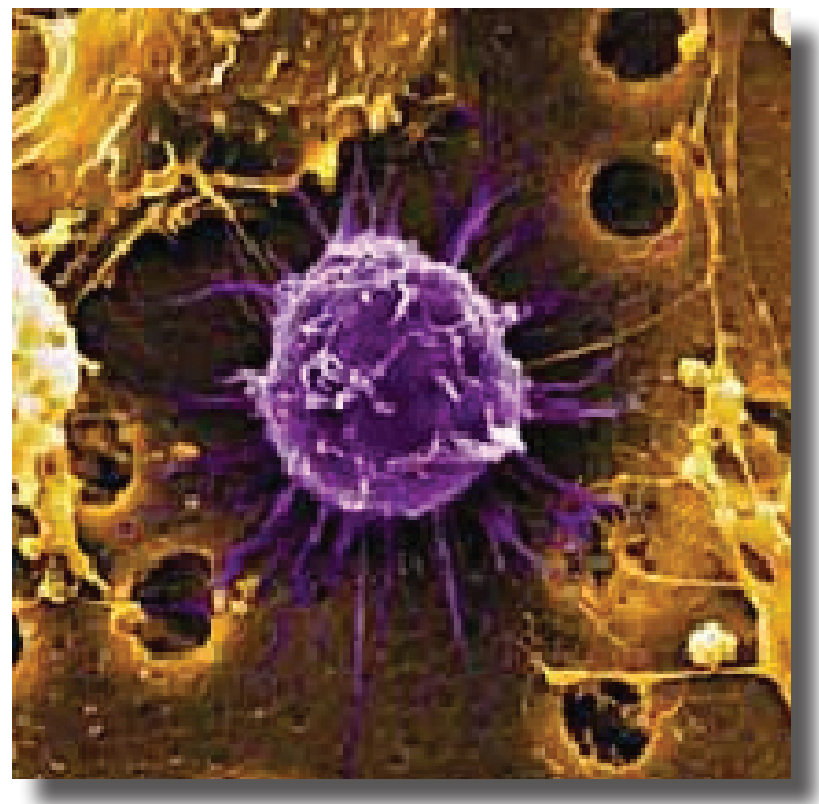

Figura 7. Célula-tronco vista através de microscopia eletrônica

Vários são os estudos sobre esse tema em andamento no mundo e o Instituto do Coração do Hospital das Clínicas da Faculdade de Medicina da venientes do próprio paciente são preparadas com estimulantes de proliferação e são colocadas no miocárdio (Figura 7). Foram estudadas 3 vias para o implante destas células: injeção direto nas coronárias por via percutânea; injeção no miocárdio por via percutânea; e injeção direta no miocárdio. A injeção direta no miocárdio parece apresentar os melhores resultados (Figura 8 ).

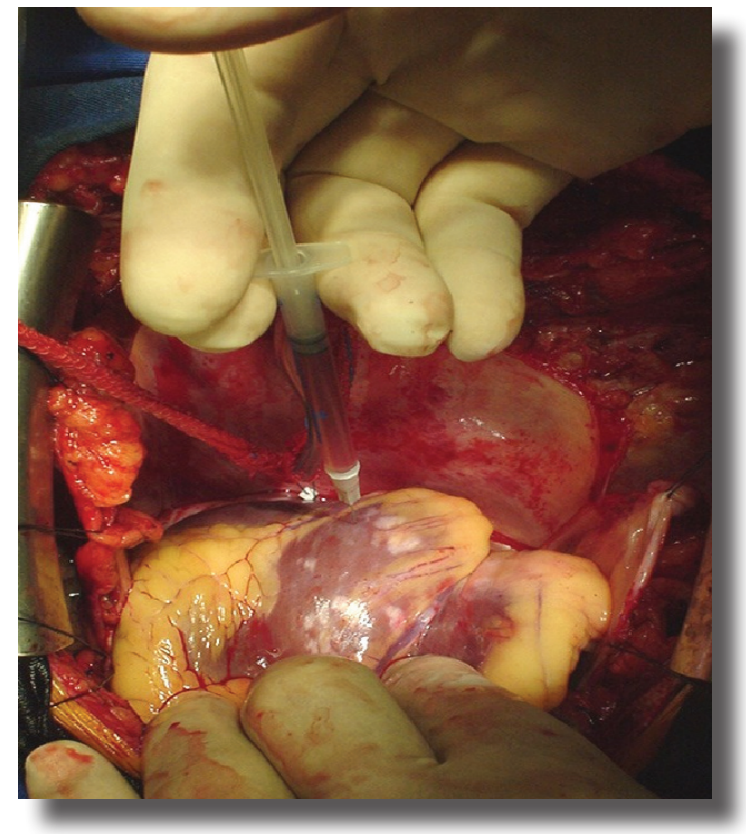

Figura 8. Injeção de células diretamente no miocárdio
Universidade de São Paulo é centro de referência internacional na pesquisa de neoangiogênese, atualmente.

Pego-Fernandes PM, Gaiotto FA, Guimarães-Fernandes F. Coronary artery bypass graft: state of the art. Rev Med (São Paulo). 2008 abr.-jun.;87(2):92-8.

ABSTRACT: The atherosclerotic cardiovascular disease is the greatest cause of death in Brazil as much as in the developed world. Therefore, it's a relevant issue for health professionals and for general population. So, we present the treatments for the disease, focusing the surgery, with revascularization of the myocardium. Then, we start with a short report that shows how the operation achieves its actual state, the techniques currently applied in this kind of operation and when it's done with or without extracorporeal circulation; who the patients for this treatment are and what results we can expect; the perspectives for this procedure, such as the practice of minimally invasive surgeries and the utilization of robotics to help the surgeon. At last, we contextualize two new procedures: the percutaneous revascularization, that still shows uncertain long-term results, and the neoangiogenesis, which still is in study phase.

KEY WORDS: Myocardial revascularization/trends. Myocardial ischemia/surgery. Surgical procedures, minimally invasive. 


\section{REFERÊNCIAS}

1. Angelini GD, Taylor FC, Reeves BC, Ascione R. Early and midterm outcome after off-pump and onpump surgery in beating heart against cardioplegic arrest studies (BHA - CAS 1 and 2): a pooled analysis of two randomized controlled trials. Lancet 2002;359:1194-9.

2. Goldman L, Ausiello D, editores. Cecil, tratado de medicina interna. Rio de Janeiro: Elsevier; 2005. 2v.

3. Camenzind E. Treatment of in-stent restenosis - Back to the future? N Engl J Med. 2006;355:2149-51.

4. Frank W, Ruel M. Vascular growth factors and angiogenesis in cardiac surgery Ann Thorac Surg. 2003;75:S685-90.

5. Kouchoukos NT, Hanley FL, Doty DB, Karp RB, Blackstone $\mathrm{EH}$, editors. Kirklin/Barratt-Boyes cardic surgery: morphology, diagnostic criteria, natural history, techniques, results, and indications. 3 rd ed. Philadelphia Elsevier Health Sciences;2003. v.3.

6. Loop FD, Litle BW, Cosgrove DM, Stewart RW, Goormastic M, Willians GW, et al. Influence of the internal mammary artery graft on 10-year survival and other cardiac events. N Engl J Med. 1986;314:1-6

7. Lytle BW, Blackstone EH, Loop F, Houghtaling PL, Arnold $\mathrm{JH}$, Akhrass $\mathrm{R}$, et al. Two internal thoracic artery are better than one. J Thorac Cardiovasc Surg. 1999;117:855-72.

8. Brasil. Ministério da Saúde. IBGE - DATASUS. 2005.

9. Puskas JD, Williams WH, Mahoney EM, Huber PR, Block PE, Duke PG et al. Off-pump vs conventional coronary artery bypass grafting: early and 1-year graft patency, cost, and quality-of-life outcomes: a randomized trial. JAMA. 2004;291:1841-9.

10. Favaloro RG. Saphenous vein autograft replacement of severe segmental coronary artery occlusion: operative technique. Ann Thorac Surg. 1968;5:334-9.

11. Mueller RL, Rosengart TK, Isom OW. The history of surgery for ischemic heart disease. Ann Thorac Surg. 1997;63:869-78.

12. Souza AGMR, Mansur AJ, editores. SOCESP Cardiologia. Sociedade de Cardiologia do Estado de São Paulo. São Paulo: Atheneu; 1996.

13. Virmani R, Farb A, Guagliumi G, Kolodgie F. Drugeluting stents: caution and concerns for long-term outcome. Cor Art Dis. 2004;6:313-8.

14. Hueb W, Gersh BJ, Costa F, Lopes N, Soares PR, Dutra $P$, et al. Impact of diabetes on five-year outcomes of patients with multivessel coronary artery disease Ann Thorac Surg. 2007;83:93-9. 